\title{
Comparing the Effect of Topical Anesthesia and Retrobulbar Block With Intravenous Sedation on Hemodynamic Changes and Satisfaction in Patients Undergoing Cataract Surgery (Phaco Method)
}

\author{
Soudabeh Haddadi ${ }^{1,}$; Shideh Marzban ${ }^{1}$; Baharak Fazeli ${ }^{1}$; Abtin Heidarzadeh ${ }^{1}$; Arman \\ Parvizi ${ }^{1}$; Bahram Naderinabi ${ }^{1}$; Mohamad Reza Panjtan Panah ${ }^{1}$ \\ ${ }^{1}$ Anesthesiology Research Center, Faculty of Medicine, Guilan University of Medical Sciences, Rasht, Iran \\ ${ }^{*}$ Corresponding author: Soudabeh Haddadi, Anesthesiology Research Center, Faculty of Medicine, Guilan University of Medical Sciences, Rasht, Iran. Tel: +98-9111323739, E-mail: \\ So_haddadi@yahoo.com
}

Received: October 30, 2014; Revised: December 1, 2014; Accepted: December 17, 2014

Background: Cataract is one of the most common surgical procedures in the elderly. In most cases, the elderly have cardiac ischemia or chronic coronary diseases, which would lead to more ischemic events during general anesthesia. Therefore, surgeons and anesthetists prefer regional aesthesia to the general one owing to its more advantages and less complications.

Objectives: Therefore, this study aimed to compare topical method and retrobulbar block for pain intensity, patient's satisfaction, hemodynamic changes and intra and postoperative complications.

Patients and Methods: In a single-blinded clinical trial, 114 patients scheduled for cataract surgery, aged 50 to 90 years with ASA physical status of I-III, were randomly assigned to two groups under monitored anesthesia care as topical anesthesia and retrobulbar block. After the injection of intravenous sedation, which was the combination of midazolam 0.5-1 mg with fentanyl 0.5-1 $\mu / \mathrm{kg}$, patients received retro bulbar block or topical anesthesia. During the operation, heart rate, systolic and diastolic blood pressure, mean arterial blood pressure and arterial saturation of O2were measured every five minutes. In addition, pain (VAS) and satisfaction (ISAS) scores were recorded every 15 minutes, then at recovery and one hour after the ending of operation in the ward. Findings were statistically analyzed using SPSS 16. Results: In this study, no significant association was found between age, gender, education and physical condition of patients in both topical and retro bulbar block groups. Comparison of pain based on VAS, satisfaction based on ISAS score and MAP in the studied periods had no significant differences between the two groups of patients undergoing cataract surgery. However, significant differences were found between the two groups $(\mathrm{P}=0.045,0.02,0.042$ and $\mathrm{P}<0.05)$ regarding heart rate, systolic and diastolic blood pressure and arterial oxygen saturation percentage after $20-30$ minutes of the operation.

Conclusions: Both methods, topical and retro bulbar block had similar impression in cataract surgery regarding analgesia and patient satisfaction. However, in non-complicated cataract surgeries with short duration, topical anesthesia may be the preferable method, because of non-invasiveness, appropriate analgesia, patient satisfaction and hemodynamic stability.

Keywords: Cataract; Retro Bulbar Block; Topical; Anesthesia; Hemodynamics; Variables; Pain; Satisfaction

\section{Background}

Lens opacity in cataract disturbs vision, differentiation sensitivity and light perception. With aging, its incidence rate increases and reaches $100 \%$ at 90 years $(1,2)$. Therefore, cataract surgery is the most common surgery in the elderly $(3,4)$. Cataract is a major cause of blindness worldwide (5). Since most these patients are the elderly with the risk factors of cardiac ischemia or chronic coronary disease $(6,7)$ such as old age, atherosclerosis, hypertension and diabetes mellitus, general anesthesia would lead to more cardiac ischemic events in them; also, changes in heart rate and hemodynamic instability are observed in these patients $(8,9)$.

Regional (local) anesthesia has many advantages over general anesthesia, induces better postoperative analgesia, causes less nausea and vomiting and leads to earlier patient discharge. Most patients have the standards of recovery discharge after the operation and could leave the hospital without admission in the recovery $(7,8)$. Although in our academic hospital, patients stayed postoperative 24 hours in the ward.

Eye surgery requires eye immobilization and deep anesthesia of the surgical site, which can be safely, performed using regional anesthesia (6). Among all the regional anesthetic manners, two methods were selected in this study; one was retro bulbar injection of local anesthetics and the other was topical use of anesthetic drops.

\section{Objectives}

With searching previous studies, we decided to com- 
pare retrobulbar block with topical method for intraand postoperative pain, changes of hemodynamic parameters and patient satisfaction in Amir-Al-Momenin Academic Hospital, Rasht, Iran. Assuming that topical method is non-invasive and induces similar analgesia and anesthesia induced by retrobulbar injection, it may be a better alternative among regional methods (10-13), with least pain and hemodynamic changes.

\section{Patients and Methods}

This single-blinded clinical trial was performed from 2013-2014 after approval of the project proposal by the Vice Chancellor for Research and Technology of Guilan University of Medical Sciences and Ethics Committee and registration in the Iranian Registry of Clinical Trials (IRCT2013122815963N1). After explanation of study issues and fulfilling the informed consent, the study was conducted on 114 patients scheduled for cataract surgery aged 50 to 90 years with ASA physical status I-III in AmirAl-Momemnin Hospital, Rasht, Iran. Exclusion criteria were coagulopathy, using antiplatelet drugs, tremor in head (Parkinson), cognitive disorders, Alzheimer, claustrophobia, chronic spontaneous cough (10) opium addiction, chronic analgesic usage and allergy to local anesthetics. Whenever the operation time was prolonged more than one hour or while general anesthesia was induced, these subjects were excluded. The study was organized in a single blinded design (the assessor did not know about the anesthesia method); however, the surgeon and anesthesiologist were aware to take required measures in case of unfavorable complications.

First, all demographic information including gender, age and education were recorded by a colleague in special forms. Then, patients were randomly assigned (with random fixed block) to one of the two groups of topical anesthesia and retro bulbar block. In the both groups, patients received intravenous sedation contained $0.5-1 \mathrm{mg}$ of midazolam plus $0.5-1 \mathrm{mic} / \mathrm{kg}$ body weight of fentanyl (depending on the patients' physical conditions). Group $\mathrm{R}$ underwent retro bulbar block by an ophthalmologist using $2 \mathrm{~mL}$ of Lidocaine $2 \%$ and hyaluronidase 1/15000 with a 23-gauge needle with a length of $3 \mathrm{~cm}$.

The needle was entered the eye right at the junction of lower and outer edges of the eye, just above the lower eye edge and pushed inside until being placed between extra-ocular muscles. Then, $2 \mathrm{~mL}$ of the anesthetic solution was injected. In group T, after administering intravenous sedation, 2 to 3 drops of tetra Caine $0.5 \%$ were poured into the eyes. This was repeated maximum up to 3 times with 5 minutes intervals. During the operation, electrocardiography (lead 2), blood pressure and arterial oxygen saturation were monitored and oxygen with nasal cannula with a rate of $5 \mathrm{~L} / \mathrm{min}$ was provided for the patient. Interoperation, patient's heart rate, systolic and diastolic blood pressure, mean arterial blood pressure and arterial oxygen saturation were checked every five minutes.
In addition, pain and satisfaction scores were recorded every 15 minutes. The surgical period was 30 to $45 \mathrm{~min}$ utes. Information was also recorded after the operation in the recovery and one hour after the transmission in the ward. Ephedrine was prescribed in case of hypotension (decreased BP of more than $20 \%$ of the basal BP) and atropine was prescribed in case of bradycardia (HR of less than 60 beat/min or 50 beat/min in beta-blocker users), respectively (11). Intraoperative hypertension (BP > 160/90 mmHg) was treated with TNG $5 \mu / \mathrm{min}$.

In patients with diabetes, Fasting Blood Sugar between $120-180 \mathrm{mg} / \mathrm{dL}$ was acceptable. FBS was checked in the early morning of operation and repeated related to patients' condition and level of BS (on average every 2 hours).Blood Sugar less than $50 \mathrm{mg} / \mathrm{dL}$ (in alert subjects) was corrected with four sugar cube, interoperation with $0.5 \mathrm{~g} / \mathrm{kg}$ intravenous dextrose. Patients with hyperglycemia (FBS > 250) were treated with insulin based on chart (12).

Finally, collected data was analyzed using statistical software (SPSS ver. 16, SPSS Inc. Chicago, IL, USA).Chi square and t test was used for data analysis. P value less than 0.05 was considered significant.

\begin{tabular}{lccc}
\hline Box 1. VAS and ISAS Classifications & \multicolumn{2}{l}{} \\
\hline \multicolumn{2}{l}{ VAS (Visual Analog Scale) } & \multicolumn{2}{l}{$\begin{array}{l}\text { ISAS (Iowa Satisfaction } \\
\text { With Anesthesia Scale) }\end{array}$} \\
\hline $\mathbf{0}$ & No pain & 1 & Weak \\
$\mathbf{2}$ & Mild & 2 & Average \\
$\mathbf{4}$ & Average & 3 & Good \\
$\mathbf{6}$ & Strong & 4 & Very good \\
$\mathbf{8}$ & Very strong & 5 & Excellent \\
$\mathbf{1 0}$ & Strongest form & 6 & Perfect \\
\hline
\end{tabular}

\section{Results}

The study was conducted on 114 patients aged 50-90 years who were candidates for cataract surgery from 2013-2014 in Amir-Al-Momenin Academic Hospital. The patients were randomly divided into two groups of retro bulbar block and topical anesthesia and were observed in time intervals (intraoperative), recovery and one hour post-op in the ward for pain intensity, patient satisfaction and hemodynamic parameters. Patient characteristics were described in Table 1. Based on Chi-square test, no significant association was found between age, gender, education and physical condition of patients between topical and retrobulbar block groups $(\mathrm{P}>0.05)$ (Table 1$)$.

Comparing pain score based on VAS scale in different time periods using Mann-Whitney U, there was no significant difference between the two groups of patients undergoing cataract surgery using topical or retro bulbar blocks (>0.05) (Table 2 and Figure 1 ). 
Haddadi S et al.

\begin{tabular}{|c|c|c|c|c|}
\hline Variables & Topical Anesthesia & Retro Bulbar Block & Total & P Value \\
\hline Age, $y$ & & & & 0.171 \\
\hline$<60$ & $10(17.5)$ & $16(28.1)$ & $26(22.8)$ & \\
\hline $61-70$ & $17(29.8)$ & $17(29.8)$ & $34(29.8)$ & \\
\hline $71-80$ & $24(42.1)$ & $14(24.6)$ & $38(33.3)$ & \\
\hline$>80$ & $6(10)$ & $10(17.5)$ & $16(14)$ & \\
\hline Education & & & & 0.21 \\
\hline Illiterate-primary school & $49(86)$ & $44(77.2)$ & $93(81.6)$ & \\
\hline Under high school & $6(10.5)$ & $6(10.5)$ & $12(10.5)$ & \\
\hline High school graduate & $2(3.5)$ & $7(12.3)$ & $9(7.9)$ & \\
\hline ASA & & & & 0.83 \\
\hline I & $10(17.5)$ & $11(19.3)$ & $21(18.4)$ & \\
\hline II & $38(66.7)$ & $35(61.4)$ & $73(64)$ & \\
\hline III & $9(15.8)$ & $11(19.3)$ & $20(17.5)$ & \\
\hline Gender & & & & 0.849 \\
\hline Male & $23(40 / 4)$ & $24(42 / 1)$ & $47(41 / 2)$ & \\
\hline Female & $34(59.6)$ & $33(57.9)$ & $67(58.8)$ & \\
\hline
\end{tabular}

${ }^{\mathrm{a}}$ All of the values are presented as No.(\%).

Table 2. Comparing Pain Intensities Based on VAS Scale in Time Intervals in Two Groups of Cataract Surgery (Phaco) Using Topical A and Retrobulbar B

\begin{tabular}{|c|c|c|c|c|}
\hline Time, min & Number & Mean \pm SD & Z Value & PValue \\
\hline Beginning of operation & & & 1.15 & 0.25 \\
\hline Topical & 57 & $0.07 \pm 0.37$ & & \\
\hline Retro bulbar & 57 & $0.15 \pm 0.52$ & & \\
\hline $15^{\text {th }}$ & & & 1.38 & 0.165 \\
\hline Topical & 57 & $0.21 \pm 0.61$ & & \\
\hline Retro bulbar & 57 & $0.61 \pm 1.5$ & & \\
\hline $30^{\text {th }}$ & & & 1.12 & 0.26 \\
\hline Topical & 30 & $0.26 \pm 0.69$ & & \\
\hline Retro bulbar & 48 & $0.66 \pm 1.44$ & & \\
\hline $45^{\text {th }}$ & & & 0.097 & 0.92 \\
\hline Topical & 9 & $1.33 \pm 2.64$ & & \\
\hline Retro bulbar & 19 & $0.52 \pm 0.9$ & & \\
\hline In recovery & & & 0.41 & 0.67 \\
\hline Topical & 57 & $0.4 \pm 0.77$ & & \\
\hline Retro bulbar & 57 & $0.52 \pm 0.96$ & & \\
\hline In ward & & & 1.74 & 0.082 \\
\hline Topical & 57 & $0.22 \pm 0.62$ & & \\
\hline Retro bulbar & 57 & $0.49 \pm 0.86$ & & \\
\hline
\end{tabular}




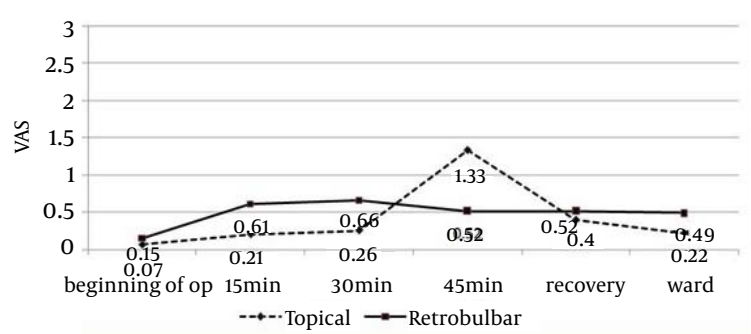

Figure 1. Comparing Pain Intensities Based on VAS Scale in Time Intervals in the Two Groups of Cataract Surgery (Phaco) Using Topical Anesthesia and Retrobulbar Block

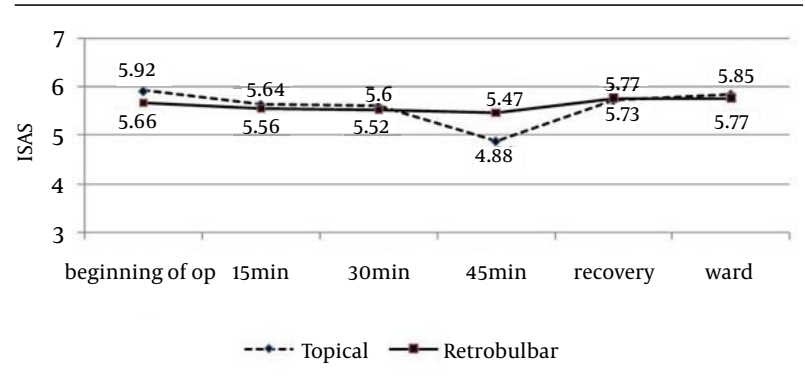

Figure 2. Comparing Patient Satisfaction Based on ISAS Scale in Time Intervals in the Two Groups of Cataract Surgery (Phaco) Using Topical Anesthesia and Retrobulbar Block

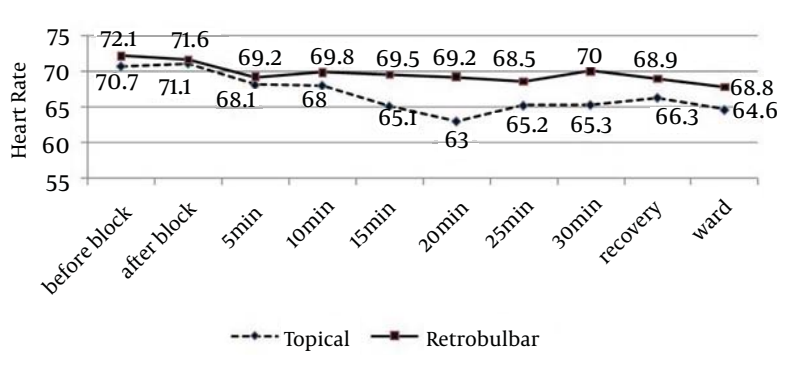

Figure 3. Comparing Heart Rate of Patients in Time Intervals in the Two Groups of Cataract Surgery (Phaco) Using Topical Anesthesia and Retrobulbar Block

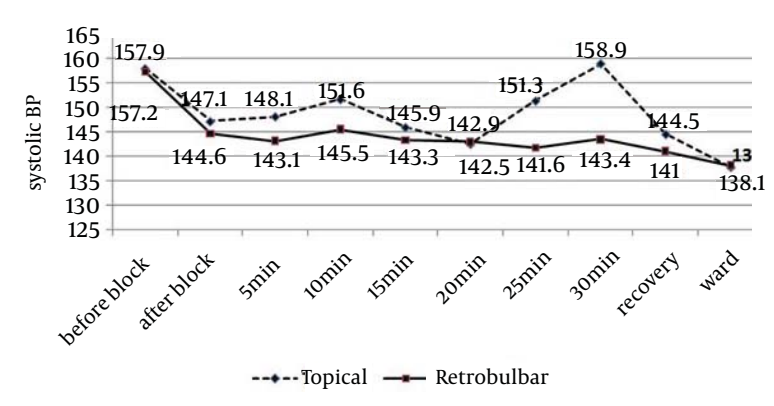

Figure 4. Comparing Systolic Blood Pressure of Patients in Time Intervals in the Two Groups of Cataract Surgery (Phaco) Using Topical Anesthesia and Retrobulbar Block

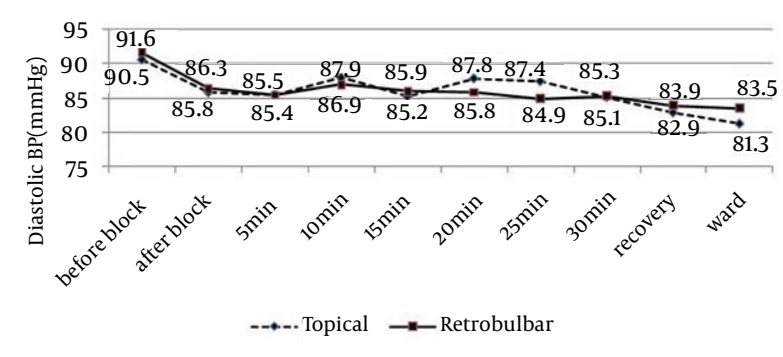

Figure 5. Comparing Diastolic Blood Pressure of Patients in Time Intervals in the Two Groups of Cataract Surgery (Phaco) Using Topical Anesthesia and Retrobulbar Block

Comparing patient satisfaction based on the ISAS score at different time intervals (beginning of surgery, $15^{\text {th }}$, $30^{\text {th }}$ and $45^{\text {th }}$ minutes, recovery and in the ward) for the two cataract surgery groups of topical anesthesia and retro bulbar block, no significant differences were observed in any time periods $(\mathrm{P}>0.05)$ (Figure 2).

Furthermore, comparing hemodynamic parameters including heart rate, systolic blood pressure, diastolic blood pressure and mean arterial blood pressure between patients undergoing cataract surgery using topical anesthesia and retro bulbar block, no statistically significant differences (Mann Whitney U test) were observed in any time intervals $(\mathrm{P}>0.05)$. However, there was a significant difference (General Linear Model, ANCOVA) for heart rate 20 minutes after the block with the confidence of $95 \%$ and error of less than $5 \%(P<0.045)$ (Figure 3$)$.

Comparing systolic blood pressure demonstrated no significant difference between the two groups $(\mathrm{P}>0.05)$; however, there was a significant difference 30 minutes after the block (Mann Whitney U test, General Linear Model, ANCOVA) $(\mathrm{P}=0.02)$ (Figure 4). For diastolic blood pressure, no significant difference was observed between the two groups, except 20 minutes after topical anesthesia, at which a significant difference was found between the two groups (Mann Whitney U test, General Linear Model, ANCOVA) $(\mathrm{P}=0.042)$ (Figure 5). Regarding mean arterial pressure, there was no significant difference at different times (Two-independence sample test, General Linear Model, ANCOVA) (P>0.05).

Comparing arterial oxygen saturation percentage between the two cataract surgery groups, there was no significant difference between topical anesthesia and retro bulbar block, except for the periods after the block and $5^{\text {th }}$ and $10^{\text {th }}$ minutes, at which significant differences were observed (Mann Whitney U test, General Linear Model, ANCOVA) $(\mathrm{P}=0.008,0.02$ and 0.029). No patient's anesthetic plan was changed to general anesthesia in the both groups. No intra- and postoperative complications including nausea, vomiting or reduced arterial oxygen saturation were found.

\section{Discussion}

In this randomized single-blinded clinical study, effects 
of topical anesthesia and retro bulbar block on hemodynamic changes, pain and satisfaction of patients undergoing cataract surgery were investigated. Midazolam 0.5$1 \mathrm{mg}$ with Fentanyl 0.5-1 $\mathrm{mic} / \mathrm{kg}$ was used to reduce pain in patients $(1,10,13)$.

There were no statistically significant differences regarding pain intensity based on VAS scale and patient satisfaction based on ISAS between the two groups. Although, when the operation under topical anesthesia lasted nearly 30 minutes, hemodynamic changes occurred (increasing SBB, DBP and decreasing HR).

Cataract surgery is the most common ophthalmic surgical procedure performed in the elderly. Regional anesthesia under monitored anesthesia care is often the preferred method for the elderly, because of their high age and underlying cardiopulmonary diseases (1-7).

During the operation under MAC (Monitored Anesthesia Care), patients were fully monitored by an anesthesiologist and to reduce pain anxiety and patient comfort, they were given drugs during block (10,14-17). There are numerous regional anesthetic methods for cataract surgery in different studies $(6-9,18,19)$. Some studies demonstrated the preference of regional to topical anesthesia.

In Ryu's study, three methods of topical, retro bulbar and sub-Tenon were compared and it was found that mean arterial pressure and heart rate in the retro bulbar group were considerably higher than topical and subTenon groups during and immediately after the block. On the other hand, in the sub-Tenon group, patient satisfaction was the maximum (1). In our study, there was no significant difference in patient satisfaction and pain intensity; only when topical surgery lasted for more than nearly 30 minutes, reduced satisfaction $(P=0.92)$, increased pain $(\mathrm{P}=0.267)$ and hemodynamic changes $(\mathrm{P}<$ $0.05)$ were resulted.

Boezaart et al. conducted a study on 98 patients who underwent cataract surgery in one eye in two topical or mixed retro bulbar and peri-bulbar combination groups. One week later, the operation of the opposite eye was performed in a different group from the previous operation (first operation using topical was performed by block method in the second surgery). Results showed that retro bulbar and peri-bulbar groups had more satisfaction and topical group had more difficult operating conditions (7). However, in the present study, there was no significant difference for satisfaction between the two topical and retro bulbar groups; although when surgery under topical anesthesia lasted more than 30 minutes, reduced satisfaction and pain intensity were resulted (no statistical significant).

Balkan et al. conducted a study on 191 patients undergoing cataract surgery who received midazolam and fentanyl and found no significant difference in pain and need for sedative drugs, which was in line with the results of the present study (8).

Jacobi et al. performed a study on 476 patients scheduled for cataract surgery using phaco emulsification techniques under retro bulbar anesthesia with bupivacaine $0.75 \%$, lidocaine $2 \%$, and hyaluronidase or topical anesthesia with lidocaine $2 \%$. Surgical complications, intraoperative situation and pain intensity were evaluated based on VAS. Except the incidence of vitreous loss, which was lower in the topical group, there were no significant differences in other complications between the groups. Pain score was not significantly different between the two groups. Patients had more tendency to use topical than retro bulbar anesthesia $(\mathrm{P}=0.01)$. For the surgeons, the operation was more difficult in the topical than retro bulbar groups (6). In this study, there was no statistically significant difference for pain, satisfaction and complications between the groups.

However, Nwosu et al. investigated two retro bulbar and sub conjunctival methods in 90 patients undergoing cataract surgery. The patients were evaluated for pain, eye immobilization and postoperative ptosis. Both of these techniques were safe and effective for cataract surgery; however, the pain experienced by patients in retro bulbar group was slightly more (2). In the current study, there was no statistically significant difference in pain between the two groups.

Sauder compared two topical and peri-bulbar techniques in cataract surgery and reported no significant difference in pain, surgical complications and postoperative visual outcomes between these two groups (20). Similarly, in this study, there was no difference between topical and retro bulbar groups regarding pain intensity. Gombos et al. compared topical and retro bulbar block in cataract surgery on 115 patients and reported that retro bulbar group experienced less pain and discomfort and SBP was significantly lower (21), but in our study there was no difference between topical and retro bulbar groups for pain intensity and satisfaction. Only when topical operation lasted for more than nearly 30 minutes, reduced satisfaction $(P=0.92)$, increased pain $(P=0.267)$ and hemodynamic changes $(\mathrm{P}<0.05)$ were resulted.

In a 50-year study on 19250 patients undergoing cataract surgery in 90 centers, Katz et al. studied surgeries using topical, block and sedating drugs and investigated patients regarding intraoperative pain, satisfaction with pain control and postoperative complications (nausea, vomiting and dizziness). Intraoperative pain and postoperative complications were lower in the topical block group (3). Indeed, in the present study, no differences existed regarding pain between these groups.

In this investigation, most patients aged between 71 and 80 years with low education or even illiteracy and ASA class III. Comparing hemodynamic changes between the two retro bulbar and topical groups demonstrated that systolic and diastolic blood pressure evidently increased and heart rate decreased 20 and 30 minutes after the operation in topical group. Moreover, 45 minutes after the block, VAS score increased in the topical group; at the same time, satisfaction rate of patients decreased in this group (statistical non-significant). 
Arterial oxygen saturation was not greatly different between the two groups. In sum, there was no significant difference for pain intensity and satisfaction between the two groups; only when the operation lasted for more than nearly 30 minutes, satisfaction was decreased and pain and hemodynamic changes increased (statistical nonsignificant). Intraoperative and postoperative complications were not observed in recovery room and the ward. Hemodynamic changes after nearly 30 minutes may be due to decreasing the efficacy of topical drops and Occulo Cardiac Reflex (decreased HR). Although in retro bulbar block, the eye becomes immobilized providing better surgical condition and patient satisfaction than topical anesthesia, but when we studied it, there was no significant difference. Therefore, both methods, topical and retro bulbar block seem to have similar impression in cataract surgery regarding analgesia and patient satisfaction. Nevertheless, in non-complicated cataract surgeries with short duration, topical anesthesia may be the preferable method, because of non-invasiveness, appropriate analgesia, patient satisfaction and hemodynamic stability.

\section{Acknowledgements}

This research was the result of a residency thesis. Hereby, we acknowledge the deputy Vice-Chancellor for research and technology affairs of Guilan University of Medical Sciences.

\section{Authors' Contributions}

Study concept and design: Haddadi, Marzban and Fazeli; Collection of data: Haddadi, Marzban, Fazeli and Panjtan Panah; Analysis and interpretation of data: Heidarzadeh; Drafting of the manuscript: Haddadi, Parvizi and Naderinabi; Critical revision: Haddadi, Marzban, Parvizi and Naderinabi.

\section{Funding/Support}

Guilan University of Medical Sciences, Vice-Chancellor for research and technology.

\section{References}

1. Ryu JH, Kim M, Bahk JH, Do SH, Cheong IY, Kim YC. A comparison of retrobulbar block, sub-Tenon block, and topical anesthesia during cataract surgery. Eur J Ophthalmol. 2009;19(2):240-6.
2. Nwosu SN, Nwosu VO, Anajekwu C, Ezenwa A. Retrobulbar versus subconjunctival anesthesia for cataract surgery. Niger J Clin Pract. 2011;14(3):280-3.

3. Katz J, Feldman MA, Bass EB, Lubomski LH, Tielsch JM, Petty BG, et al. Adverse intraoperative medical events and their association with anesthesia management strategies in cataract surgery. Ophthalmology. 2001;108(10):1721-6.

4. Kallio H, Rosenberg PH. Advances in ophthalmic regional anaesthesia. Best Pract Res Clin Anaesthesiol. 2005;19(2):215-27.

5. Alhassan MB, Kyari F, Ejere HO. Peribulbar versus retrobulbar anaesthesia for cataract surgery. Anesth Analg. 2008;107(6):2089.

6. Jacobi PC, Dietlein TS, Jacobi FK. A comparative study of topical vs retrobulbar anesthesia in complicated cataract surgery. Arch Ophthalmol. 2000;118(8):1037-43.

7. Boezaart A, Berry R, Nell M. Topical anesthesia versus retrobulbar block for cataract surgery: the patients' perspective. J Clin Anesth. 2000;12(1):58-60.

8. Balkan BK, Iyilikci L, Gunenc F, Uzumlu H, Kara HC, Celik L, et al. Comparison of sedation requirements for cataract surgery under topical anesthesia or retrobulbar block. Eur J Ophthalmol. 2004;14(6):473-7.

9. Ruschen H, Celaschi D, Bunce C, Carr C. Randomised controlled trial of sub-Tenon's block versus topical anaesthesia for cataract surgery: a comparison of patient satisfaction. Br J Ophthalmol. 2005;89(3):291-3.

10. Marzban S, Haddadi S, Parvizi A. Anesthesia for Eye and ENT surgeries.Rasht: Gap; 2007.

11. Miller RD. Miller's Anesthesia.: Churchill Livingstone/Elsevier; 2010.

12. Hines RL, Marschall K. Stoelting's Anesthesia and Co-Existing Disease.: Elsevier Health Sciences; 2012.

13. Rewari V, Madan R, Kaul HL, Kumar L. Remifentanil and propofol sedation for retrobulbar nerve block. Anaesth Intensive Care. 2002;30(4):433-7.

14. Fung D, Cohen MM, Stewart S, Davies A. What determines patient satisfaction with cataract care under topical local anesthesia and monitored sedation in a community hospital setting? Anesth Analg. 2005;100(6):1644-50.

15. Allen D, Vasavada A. Cataract and surgery for cataract. BMJ. 2006;333(7559):128.

16. Navaleza JS, Pendse SJ, Blecher MH. Choosing anesthesia for cataract surgery. Ophthalmol Clin North Am. 2006;19(2):233-7.

17. Nouvellon E, Cuvillon P, Ripart J, Viel EJ. Anaesthesia for cataract surgery. Drugs \& aging. 2010;27(1):21-38.

18. Fang ZT, Keyes MA. A novel mixture of propofol, alfentanil, and lidocaine for regional block with monitored anesthesia care in ophthalmic surgery.J Clin Anesth. 2006;18(2):114-7.

19. Fung D, Cohen M, Stewart S, Davies A. Can the Iowa Satisfaction with Anesthesia Scale be used to measure patient satisfaction with cataract care under topical local anesthesia and monitored sedation at a community hospital? Anesth Analg. 2005;100(6):1637-43.

20. Sauder G, Jonas JB. Topical versus peribulbar anaesthesia for cataract surgery. Acta Ophthalmol Scand. 2003;81(6):596-9.

21. Gombos K, Jakubovits E, Kolos A, Salacz G, Nemeth J. Cataract surgery anaesthesia: is topical anaesthesia really better than retrobulbar? Acta Ophthalmol Scand. 2007;85(3):309-16. 